\title{
Preclinical study of patient-specific cell-free nanofiber tissue-engineered vascular grafts using 3-dimensional printing in a sheep model
}

Takuma Fukunishi, MD, ${ }^{\mathrm{a}}$ Cameron A. Best, BA, ${ }^{\mathrm{b}}$ Tadahisa Sugiura, MD, PhD, ${ }^{\mathrm{b}}$ Justin Opfermann, MS, ${ }^{\mathrm{c}}$ Chin Siang Ong, MD, ${ }^{a}$ Toshiharu Shinoka, MD, PhD, ${ }^{\mathrm{b}}$ Christopher K. Breuer, MD, ${ }^{\mathrm{b}}$ Axel Krieger, $\mathrm{PhD}$, Jed Johnson, $\mathrm{PhD},{ }^{\mathrm{d}}$ and Narutoshi Hibino, $\mathrm{MD}, \mathrm{PhD}^{\mathrm{a}}$

\begin{abstract}
Background: Tissue-engineered vascular grafts (TEVGs) offer potential to overcome limitations of current approaches for reconstruction in congenital heart disease by providing biodegradable scaffolds on which autologous cells proliferate and provide physiologic functionality. However, current TEVGs do not address the diverse anatomic requirements of individual patients. This study explores the feasibility of creating patient-specific TEVGs by combining 3-dimensional (3D) printing and electrospinning technology.
\end{abstract}

Methods: An electrospinning mandrel was 3D-printed after computer-aided design based on preoperative imaging of the ovine thoracic inferior vena cava (IVC). TEVG scaffolds were then electrospun around the 3D-printed mandrel. Six patient-specific TEVGs were implanted as cell-free IVC interposition conduits in a sheep model and explanted after 6 months for histologic, biochemical, and biomechanical evaluation.

Results: All sheep survived without complications, and all grafts were patent without aneurysm formation or ectopic calcification. Serial angiography revealed significant decreases in TEVG pressure gradients between 3 and 6 months as the grafts remodeled. At explant, the nanofiber scaffold was nearly completely resorbed and the TEVG showed similar mechanical properties to that of native IVC. Histological analysis demonstrated an organized smooth muscle cell layer, extracellular matrix deposition, and endothelialization. No significant difference in elastin and collagen content between the TEVG and native IVC was identified. There was a significant positive correlation between wall thickness and $\mathrm{CD} 68^{+}$macrophage infiltration into the TEVG.

Conclusions: Creation of patient-specific nanofiber TEVGs by combining electrospinning and 3D printing is a feasible technology as future clinical option. Further preclinical studies involving more complex anatomical shapes are warranted. (J Thorac Cardiovasc Surg 2017;153:924-32)

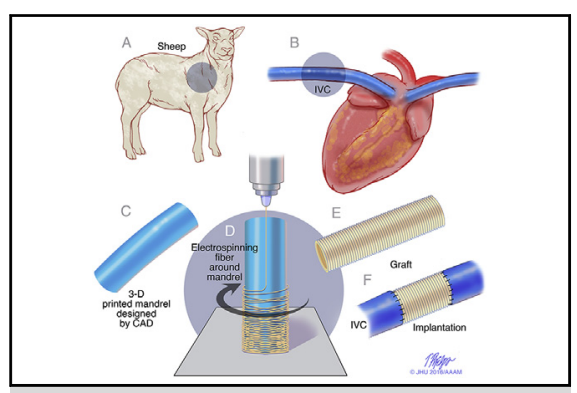

Study design of cell-free patient-specific nanofiber tissue engineered vascular graft. A and B, Designing the graft from a preoperative angiogram in the sheep model. $C$, An electrospinning mandrel was modeled by computer aided design and subsequently 3D-printed. D, The nanofiber graft was electrospun onto the 3D printed mandrel. $E$ and $F$, The graft was implanted in sheep model.

\section{Central Message}

Our integrated approach of recent progress in 3D imaging, 3D printing, and tissue engineering technology can create presurgically designed patient-specific vascular grafts.

\section{Perspective}

Despite advances in surgical management for congenital heart disease, a significant source of mortality arises from surgical complexity. Patient-specific graft design before surgery may yield benefits for quality of life. This study demonstrates our integrated approach of 3D imaging/printing, and tissue engineering technology can create presurgically designed patientspecific vascular grafts.

See Editorial Commentary page 933.

See Editorial page 923.
From the a Department of Cardiac Surgery, Johns Hopkins Hospital, Baltimore, Md; ${ }^{\mathrm{b}}$ Tissue Engineering and Surgical Research, Nationwide Children's Hospital; 'The Sheikh Zayed Institute for Pediatric Surgical Innovation, Children's National Medical Center, Washington, DC; and ${ }^{\mathrm{d}}$ Nanofiber Solutions Inc, Columbus, Ohio. This study was supported by grants from the National Institutes of Health Center for Accelerated Innovations: Technology Development Program (grant No. 1UH54HL119810-01) and Thrasher Research Fund Early Career Awards (to N.H.). Graft materials for this study were supplied by Nanofiber Solutions.

Read at the 42nd Annual Meeting of The Western Thoracic Surgical Association, Waikoloa, Hawaii, June 22-25, 2016.
Received for publication June 28, 2016; revisions received Oct 12, 2016; accepted for publication Oct 13, 2016; available ahead of print Dec 6, 2016.

Address for reprints: Narutoshi Hibino, MD, PhD, Department of Cardiac Surgery, Johns Hopkins University, 1800 Orleans St, Zayed 7107, Baltimore, MD 21287 (E-mail: nhibino1@jhmi.edu).

0022-5223/\$36.00

Copyright (C) 2016 Published by Elsevier Inc. on behalf of The American Association for Thoracic Surgery

http://dx.doi.org/10.1016/j.jtcvs.2016.10.066 


\section{Abbreviations and Acronyms \\ $3 \mathrm{D}=$ three-dimensional \\ HPF = high-power field \\ IVC = inferior vena cava \\ PGA $=$ polyglycolic acid \\ PLCL $=$ poly(L-lactide-co-e-caprolactone $)$ \\ $\mathrm{SMC}=$ smooth muscle cell \\ TCPC $=$ total cavopulmonary connection \\ $\mathrm{TEVG}=$ tissue-engineered vascular graft}

Scanning this QR code will take you to a video for the article.

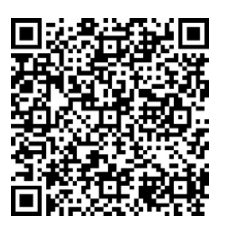

Surgical treatment of many complex congenital cardiac anomalies involves implantation of synthetic conduits of materials such as Gore-Tex and Dacron. A common example of such an application is the total cavopulmonary connection (TCPC) for single ventricle anomalies. In some instances, the use of these synthetic grafts as conduits is complicated by progressive obstruction, susceptibility to infection, and risk of thromboembolic complications. ${ }^{1,2}$ In all instances, a significant limitation to cardiovascular reconstruction is lack of growth potential of synthetic implants. For TCPC, this may lead to suboptimal management strategies, including either postponement of completion of the Fontan circulation because of patient size, or oversizing of conduits, which results in suboptimal flow characteristics, such as expiratory-phase backflow and regions of flow stagnation. ${ }^{3}$

Tissue-engineered vascular grafts (TEVGs) offer the potential to overcome these problems by providing a biodegradable scaffold in which autologous cells proliferate and mature into a physiologically functional blood vessel as scaffold polymers degrade. ${ }^{4-6}$ However, current TEVGs do not directly address the diverse anatomic and physiological requirements of individual patients.

Imaging technologies such as computed tomography and magnetic resonance imaging provide surgeons with detailed, 3-dimensional (3D) views of complex cardiovascular anatomies before surgery. We have developed novel technology to create a patient-specific TEVG using Food and Drug Administration-approved biodegradable nanofiber materials coated around a 3D printed mandrel (Figure 1).

In this study, we validated our novel technology by evaluating neotissue formation, biocompatibility, and mechanical properties of 3D-printed custom-made TEVGs after 6 months of implantation in a sheep model of high-flow, low-pressure circulation.

\section{MATERIALS AND METHODS \\ Presurgery Imaging and 3D Model/Mandrel \\ Creation}

Based on preoperative angiography images, the diameter and length of the sheep inferior vena cava (IVC) were measured and matching graft models were designed using computer-aided design software (Solidworks, Waltham, Mass). The final mandrel design was converted to stereolithography format and exported to Shapeways (New York, NY) for $3 \mathrm{D}$ fabrication out of 420 stainless steel (Figure 2).

\section{Scaffold Fabrication}

To create the co-electrospun polyglycolic acid (PGA) and poly(L-lactide-co- $\varepsilon$-caprolactone) (PLCL) scaffolds, $10 \mathrm{wt} \%$ PGA and 5 wt $\%$ PLCL were dissolved in hexafluoroisopropanol. Each solution was stirred via a magnetic stir bar for at least 3 hours at room temperature. In separate syringes, the PGA solution was dispensed at a flow rate of $2.5 \mathrm{~mL} /$ hour, and the PLCL solution was dispensed at a flow rate of $5.0 \mathrm{~mL} /$ hour to create a graft with a 1:1 PGA:PLCL ratio. Both solutions were simultaneously electrospun onto the custom 3D-printed mandrel that was positioned $20 \mathrm{~cm}$ from the needle tip and rotated at $30 \mathrm{rpm}$. A $+25-\mathrm{kV}$ charge was applied to each syringe tip, and electrospun nanofibers were deposited onto the grounded mandrel until the desired wall thickness was achieved. The electrospun scaffold was then removed from the mandrel, and the wall thickness was measured with a snap gauge by placing the scaffold between 2 glass slides. The PGA/PLCL tubes were cut into 1.5-cm lengths, and the inner diameter was $12 \mathrm{~mm}$. The scaffolds were then packaged in Tyvek pouches and terminally sterilized with gamma irradiation (Figure 2).

\section{Mechanical Testing}

Compliance and burst pressure data were acquired using a universal mechanical testing machine (MTS Systems, Eden Prairie, Minn), as described previously. ${ }^{7}$ In brief, data were acquired using a load frame fitted with a 50-pound load cell with a force resolution of $10^{-4}$ pounds and a linear displacement resolution of $10^{-8}$ inches. Compliance testing was performed using a displacement velocity of $1.5 \mathrm{~mm} /$ minute and an acquisition rate of 4 data points/second using Laplace's Law $^{8,9}$ to correlate linear force and displacement to compliance. Burst pressure testing was performed using a displacement velocity of $50 \mathrm{~mm} /$ minute and an acquisition rate of 4 data points/second using Laplace's Law ${ }^{8,10}$ to correlate linear force and displacement to burst pressure.

Ring samples were placed around 2 parallel L-shaped steel rods. One rod was attached to the base of the testing machine, and the other was attached to the load cell. The samples were strained perpendicular to the length of the sample. Compliance was calculated using systolic and diastolic pressures of $120 \mathrm{~mm} \mathrm{Hg}$ and $80 \mathrm{~mm} \mathrm{Hg}$, respectively. Burst pressure was calculated as the maximum pressure immediately preceding failure.

\section{Graft Implantation}

The Animal Care and Use Committee at Q-Test Laboratories (Columbus, Ohio) approved the care, use, and monitoring of animals for these experiments. Six custom-made cell-free nanofiber TEVGs were implanted as IVC interposition grafts in sheep (mean body weight, $23.9 \pm 5.0 \mathrm{~kg}$ ). The animal size increased by roughly 2 -fold over the 6-month study interval (mean body weight, $46.2 \pm 4.2 \mathrm{~kg}$ ). All sheep were anesthetized with $1.5 \%$ isoflurane during surgery. The IVC was exposed, and heparin (100 IU/kg) was administered intravenously. The TEVG was implanted as a supradiaphragmatic IVC interposition graft 


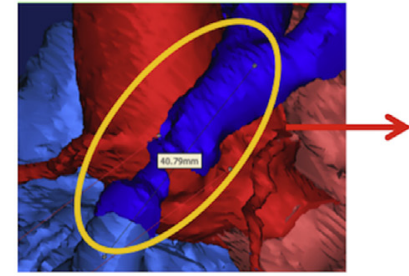

A

A

B

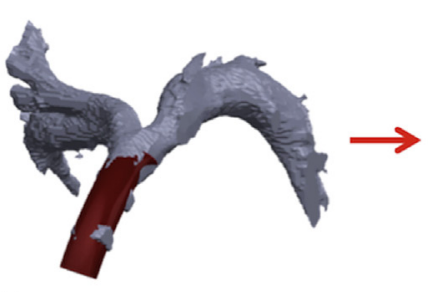

B

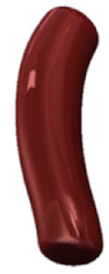

C

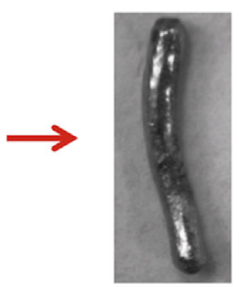

D

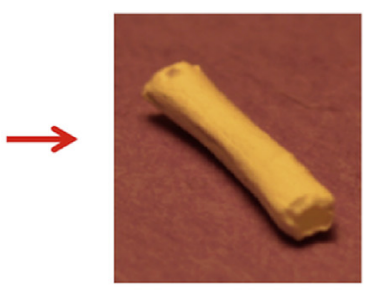

E

FIGURE 1. Flow chart depicting the proposed process for manufacturing patient-specific tissue-engineered vascular grafts. A, Segmenting 3D image of the vasculature. B, Creating a patient-specific graft design from the preoperative computed tomography image using the computer-aided design system. C, Finalized mandrel. D, 3D-printed mandrel from stainless steel. E, Patient-specific nanofiber graft.

using standard running 6-0 Prolene suture (Figure 2). Two surgical clips were placed on the proximal and distal anastomoses as reference markers for angiography and necropsy. Native supradiaphragmatic IVC served as a control group, because the sheep supradiaphragmatic IVC is longer than the human IVC, and it is located far away from right lung with no surrounding tissues. Antibiotic treatment (cefazolin) was administered intraoperatively and 7 days postoperatively. All sheep were maintained on a daily oral dose of aspirin ( $325 \mathrm{mg} /$ day) until the 6-month endpoint.

\section{Angiography}

Angiography was performed to assess any potential graft complications at the 3-and 6-month time points. A 5 Fr catheter was inserted into the jugular vein to the IVC, and intravenous contrast was manually -injected into the supradiaphragmatic IVC and midgraft. In addition, IVC blood pressure was measured at the proximal and distal anastomoses to evaluate the pressure gradients across the graft.

\section{Histology and Immunohistochemistry}

Explanted TEVG samples were fixed in $10 \%$ formalin for 24 hours at $4{ }^{\circ} \mathrm{C}$, and then embedded in paraffin. For standard histology, tissue sections were stained with hematoxylin and eosin, Masson trichrome, picrosirius red, Hart, and von Kossa stains. For immunohistochemistry, tissue sections were deparaffinized, rehydrated, and blocked for endogenous peroxidase activity and nonspecific staining. The primary antibodies used included von Willebrand factor (1:2000; Dako, Carpinteria, Calif), $\alpha$-smooth muscle actin (1:500; Dako), myosin heavy chain (1:500; Abcam, Cambridge,
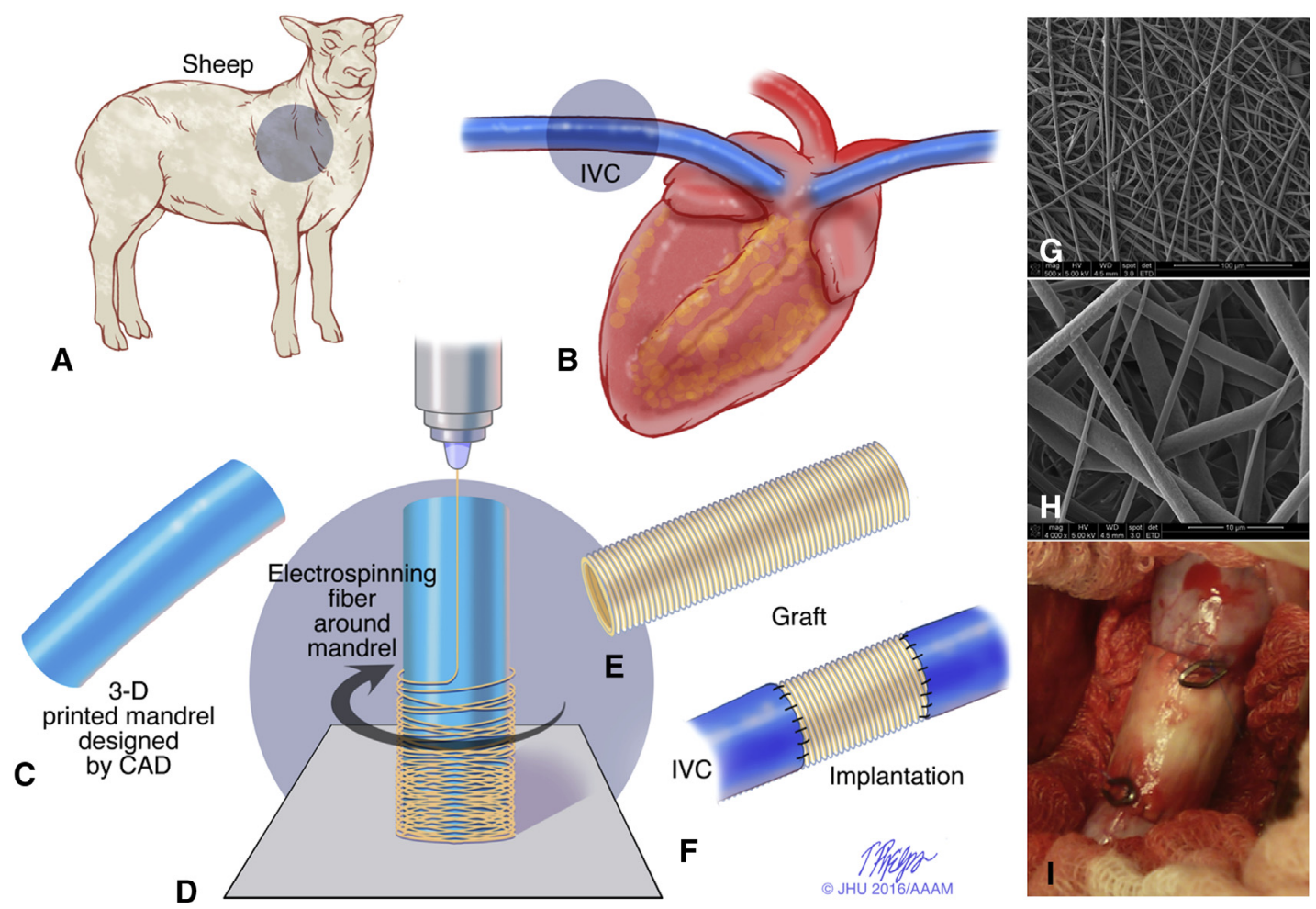

FIGURE 2. Study design of cell-free patient-specific nanofiber tissue engineered vascular graft. A and B, The dimension and shape of the thoracic inferior vena cava (IVC) was measured from angiography before surgery in the sheep model. C, An electrospinning mandrel was modeled by computer-aided design and subsequently 3D-printed. D, The nanofiber scaffold was electrospun onto the 3D-printed mandrel. E and F, A patient-specific cell-free nanofiber tissue-engineered vascular graft (TEVG) was implanted as an IVC interposition conduit in the sheep model. G and H, Scanning electron microscope images of the scaffold (G, 500×; H, 4000×). I, Intraoperative picture of the implanted TEVG. 3D, 3-dimensional. 

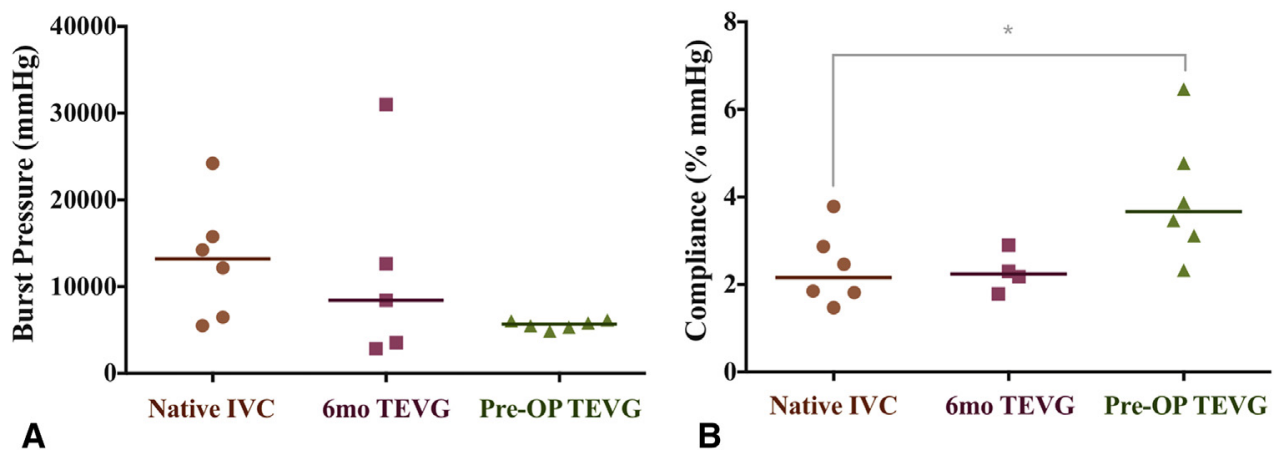

FIGURE 3. Mechanical properties. The burst pressure (A) and compliance (B) of the native inferior vena cava (IVC) and patient-specific nanofiber tissue-engineered vascular graft $(T E V G)$ both before and at 6 months after implantation. There was no significant difference in burst pressure and compliance between the native IVC and patient-specific nanofiber TEVG at 6 months. $* P<.05$.

Mass), and CD68 (1:200, Abcam). Antibody binding was detected using biotinylated secondary antibodies (Vector Laboratories, Burlingame, Calif), followed by incubation with streptavidinated horseradish peroxidase (Vector Laboratories). Development was performed by chromogenic reaction with 3,3-diaminobenzidine (Vector Laboratories). Nuclei were counterstained with Gill's hematoxylin (Vector Laboratories).

\section{Histological and Quantitative Analysis}

The lumen diameter, wall thickness, remaining scaffold area, and collagen content were measured from hematoxylin and eosin and picrosirius red (polarized light) stainings using ImageJ software (National Institutes of Health, Bethesda, Md). Picrosirius red staining revealed collagen fibers. ${ }^{11} \mathrm{CD}^{+} 8^{+}$macrophages were quantified by analyzing 4 high- power fields (HPFs; $40 \times$ ) from a representative section of each sample $(n=6)$ and averaged.

\section{Biochemical Analysis}

Elastin content was determined using a Fastin colorimetric assay (Biocolor, Carrickfergus, United Kingdom). For this assay, $100 \mathrm{mg}$ dry weight of each sample was measured and transferred to $1.5-\mathrm{mL}$ microcentrifuge tubes containing $750 \mu \mathrm{L}$ of $0.25 \mathrm{M}$ oxalic acid. The tubes were then placed on a heat block for 60 minutes at $100^{\circ} \mathrm{C}$ to convert insoluble elastin to water-soluble $\alpha$-elastin. The elastin content in each sample was determined by detection at $513 \mathrm{~nm}$ and extrapolation to a standard curve after precipitation and dye binding following the manufacturer's protocol.

Collagen content was determined by a Sircol colorimetric assay (Biocolor). For this, $100 \mathrm{mg}$ dry weight of each sample was measured and transferred to low-protein-binding $1.5-\mathrm{mL}$ conical microcentrifuge tubes containing $1.0 \mathrm{~mL}$ of pepsin (Sigma-Aldrich, St Louis, Mo), with a concentration of $0.1 \mathrm{mg} / \mathrm{mL}$ of $0.5 \mathrm{M}$ acetic acid to solubilize the collagen by means of overnight incubation. The collagen content in each sample was determined by detection at $555 \mathrm{~nm}$ and extrapolation to a standard curve after precipitation and dye binding following the manufacturer's protocol.

\section{Statistics}

For all experiments, data are represented graphically as scatterplots of individual values with a bar identifying the median; manuscript references are mean \pm standard deviation. Burst pressure, compliance, and outer diameter change data were analyzed via one-way ANOVA with Tukey's multiple-comparisons test. Pressure gradient data were analyzed using the paired 2-tailed $t$ test. Analysis of elastin, collagen, collagen area \%, and wall thickness data was performed with the unpaired 2-tailed $t$ test. The significance of any correlation between wall thickness and $\mathrm{CD} 68^{+}$ macrophages/HPF was determined by calculating the Pearson correlation coefficient. A $P$ value $<.05$ was considered statistically significant. Statistical analysis was performed using Prism version 6 (GraphPad Software, La Jolla, Calif).

\section{RESULTS}

\section{Mechanical Properties of Patient-Specific Cell-Free Nanofiber TEVGs}

Electrospinning created a tubular scaffold with a uniform wall thickness of $657 \pm 36 \mu \mathrm{m}$, significantly less than the native IVC wall thickness of $1365 \pm 476 \mu \mathrm{m}$. Scanning electron microscopy images of the scaffold are shown in Figure 2.

There was no significant difference in the burst pressure of the graft before implantation compared with native IVC. Similarly, no significant difference in burst pressure was observed between the native IVC and the TEVG at 6 months (Figure 3, $A$; native IVC, 13,062 $\pm 6847 \mathrm{~mm} \mathrm{Hg}$ vs 6-month TEVG, 11,685 $\pm 11,506 \mathrm{~mm} \mathrm{Hg}$ vs preoperative TEVG, $6167 \pm 5627 \mathrm{~mm} \mathrm{Hg} ; P=.22$ ). Preoperative graft compliance was significantly greater compared with native IVC (Figure 3, $B$; preoperative TEVG, $4.0 \% \pm 1.5 \%$ vs native IVC, $2.4 \% \pm 0.85 \% ; P<.05)$; however, there was no significant difference in compliance between native IVC and TEVG after 6 months (Figure 3, $B$; native IVC, $2.4 \% \pm 0.85 \%$ vs 6 -month TEVG, $2.3 \% \pm 0.46 \%$; $P>.05)$

\section{Biocompatibility in High-Flow Low-Pressure Venous Circulation}

Graft biocompatibility, including patency and tissue remodeling, was excellent throughout the 6-month duration of the study. All sheep survived until the study endpoint with no graft-related complications, such as stenosis, dilation, or rupture (Figure 4, A). Native IVC and the patient-specific TEVG were evaluated at 3 and 6 months postoperation for evidence of stenosis or dilation with contrast-enhanced angiography. There was no significant difference in the fold change of measured lumen diameters between 3 and 6 months when comparing the native IVC 


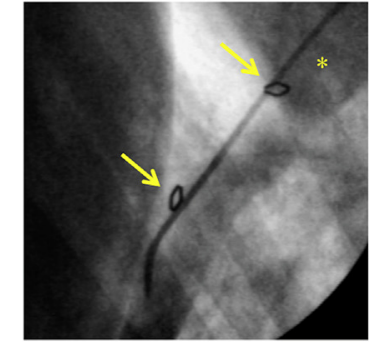

A

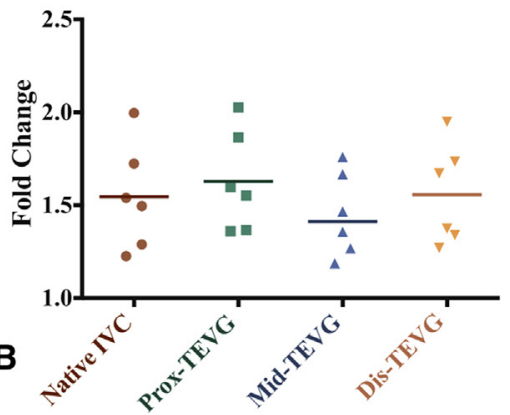

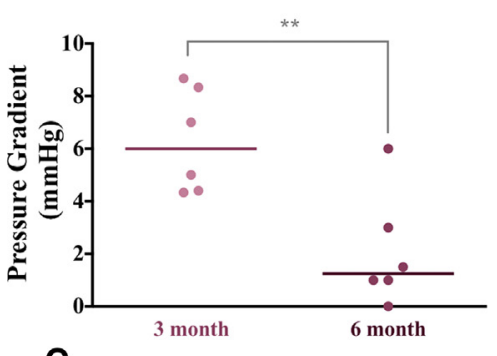

C
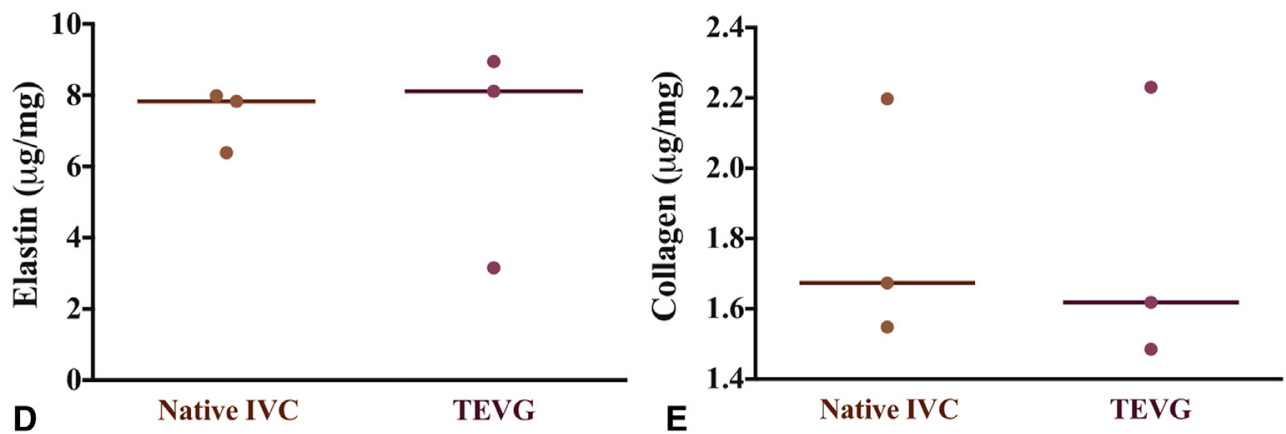

FIGURE 4. Angiographic and quantitative biochemical analyses. A, Angiography of the patient-specific nanofiber tissue-engineered vascular grafts (TEVGs) at 6 months. *Indicates right atrium; arrows indicate anastomosis site of graft. B, Angiographic diameter change over time indicating narrowing of proximal, middle, and distal regions of patient-specific nanofiber TEVGs compared with native inferior vena cava (IVC) (6M/3M diameter). C, There was a statistically significant difference in TEVG pressure gradient between the 3 - and 6-month time points. $* * P=.0045$. D and E, The elastin and collagen content of the TEVG was not significantly different from the native IVC.

with the proximal, middle, or distal regions of the TEVGs. Both the IVC and patient-specific TEVG displayed no significant diameter changes between the 3-and 6-month time points (Figure 4, $B$ ). The pressure gradient across the TEVG at 6 months was significantly less than at 3 months, suggesting advantageous remodeling and scaffold degradation (Figure 4, $C ; 3$ months, $6.3 \pm 2.0 \mathrm{~mm} \mathrm{Hg}$ vs 6 months, $2.1 \pm 2.2 \mathrm{~mm} \mathrm{Hg} ; P=.0045)$.

Elastin and collagen are critical for venous function and are well-studied markers of vascular graft remodeling. Biochemical quantification revealed the TEVG's elastin content (Figure 4, D; $6.7 \pm 3.1 \mu \mathrm{g} / \mathrm{mg}$ vs $7.4 \pm 0.88 \mu \mathrm{g} / \mathrm{mg} ; P=.74)$ and collagen content (Figure $4, E ; 1.8 \pm 0.4 \mu \mathrm{g} / \mathrm{mg}$ vs $1.8 \pm 0.35 \mu \mathrm{g} / \mathrm{mg}$; $P=.93$ ) were equivalent to that of the native ovine IVC.

\section{Well-Organized Vascular Neotissue Formation}

The tailor-made nanofiber TEVG demonstrated well-organized vascular neotissue formation over 6 months. Hematoxylin and eosin staining demonstrated extensive cellular infiltration into the TEVG, evidence that scaffold parameters, such as pore size and fiber diameter, permitted host cell infiltration (Figure 5, E). All cell-free TEVGs remained patent without complications. Importantly, hematoxylin and eosin staining visualized under polarized light microscopy revealed that only $2.09 \% \pm 0.69 \%$ of the nanofiber scaffold material remained at 6 months, indicating that the vascular neotissue was the primary contributor to the biochemical and mechanical properties assessed, further supporting the safety and efficacy of this approach. Furthermore, in vitro studies demonstrated that the TEVG loses all mechanical strength after 12 weeks (data not shown).

On the graft's luminal surface, a cellular monolayer stained positively for von Willebrand factor, confirming successful endothelialization (Figure 5, F), like native tissue (Figure $5, B$ ). The luminal surfaces displayed no evidence of microthrombosis.

Smooth muscle cells (SMCs) are important for vascular function, and we identified mature contractile vascular SMCs using $\alpha$-smooth muscle actin (Figure 5, $G$ ) and myosin heavy chain markers at 6 months (Figure $5, H$ ) in the TEVG. A layer of myosin heavy- chain positive cells was circumferentially organized and maintained adequate wall thickness at subintimal layers (Figure 5, $H$ ). A multilayered population of $\alpha$-smooth muscle actin-positive cells was present primarily in the neomedia, suggesting that the TEVG might not have been a completely mature neo-IVC at 6 months, and that active vascular remodeling was still occurring at this time point (Figure 5, G). 


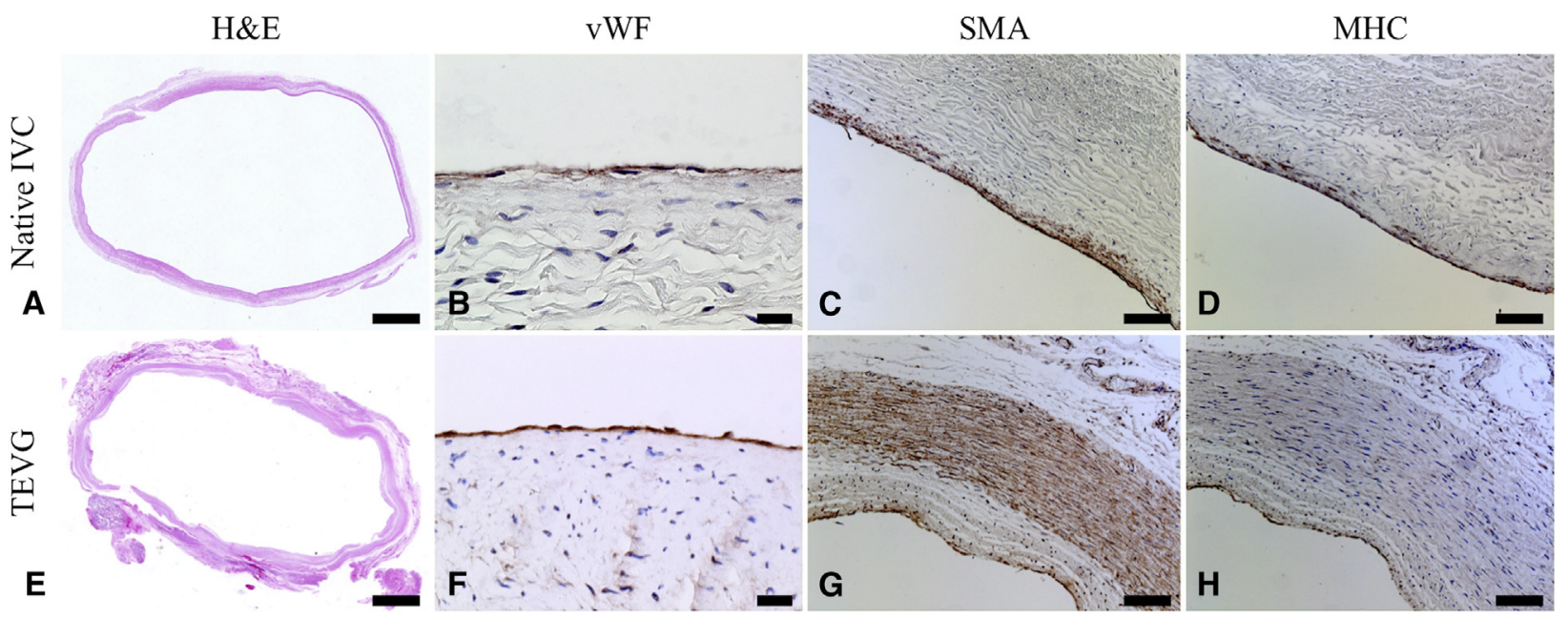

FIGURE 5. Representative hematoxylin and eosin photomicrographs of native inferior vena cava (IVC) (A) and patient-specific nanofiber tissue-engineered vascular graft (TEVG) (E). Only $2.09 \% \pm 0.69 \%$ of the nanofiber scaffold material remained at 6 months. The TEVG endothelial cells and smooth muscle cells were mature and well-organized, and the smooth muscle cell layer was thicker than the native IVC in area, distribution, and density. Representative photomicrographs are shown for von Willebrand factor (B, native IVC; F, TEVG), $\alpha$-smooth muscle actin (C, native IVC; G, TEVG), and myosin heavy chain (D, native IVC; H, TEVG). Scale bars: $2 \mathrm{~mm}$ for A and E; $20 \mu \mathrm{m}$ for B and F; $100 \mu \mathrm{m}$ otherwise.

Alternatively, $\alpha$-smooth muscle actin could indicate synthetic SMCs or myofibroblasts, and the $\alpha$-smooth muscle actin-positive/myosin heavy chain-negative staining observed in the TEVG may identify the neoadventitia or areas of continued growth and remodeling.

Extracellular matrix constituents in the TEVG mimicked the circumferential orientation observed in the native IVC. The extracellular matrix density in the TEVG (Figure 6, E, picrosirius red; $F$, Masson's trichrome) resembled that of native tissue (Figure 6, A, picrosirius red; $B$, Masson's trichrome). Collagen deposition, organization, and maturation were confirmed by assessing relative amounts and orientation of thin and thick fibers. The picrosirius red staining showed that the area fraction of collagen was similar in both the TEVG and native IVC (Figure 6, I; $9.75 \pm 6.27$ vs
$8.75 \% \pm 1.31 \% ; P=.74)$. Hart staining demonstrated that the elastin composition of the TEVG (Figure 6, G) was comparable to that of the native IVC (Figure 6, $C$ ). There was no evidence of ectopic calcification in von Kossa staining at 6 months (Figure 6, D, native IVC; $H$, TEVG).

The wall thickness of the TEVG was significantly larger than that of native IVC (Figure 7, A; TEVG $[\mathrm{n}=6]$, $2.27 \pm 0.91 \mathrm{~mm}$ vs native IVC [ $\mathrm{n}=5], 0.89 \pm 0.15 \mathrm{~mm}$; $P=.0091)$. We found a significant positive correlation between TEVG wall thickness and macrophage infiltration into the scaffold (Figure 7, B, 2-tailed Pearson correlation; $P=.0037 ; R^{2}=0.90$ ), suggesting that $\mathrm{CD}^{+} 8^{+}$macrophages induced the inflammatory process of vascular remodeling in the graft, which

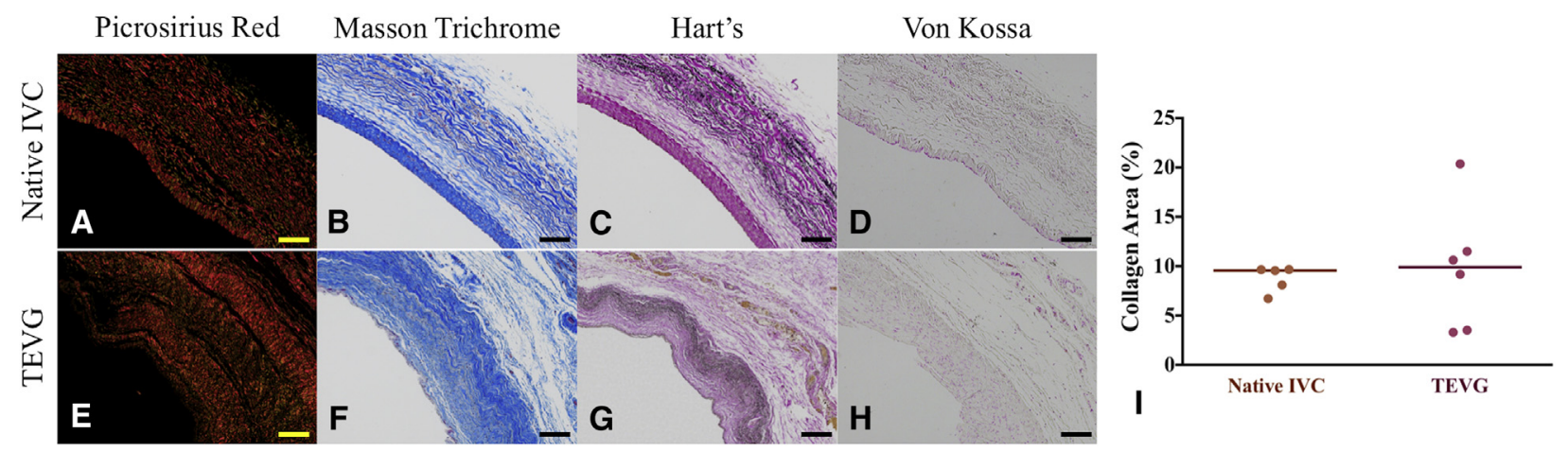

FIGURE 6. Collagen and elastin deposition in the tissue-engineered vascular graft $(T E V G)(\mathrm{E}-\mathrm{H})$ at 6 months is similar to that of native inferior vena cava (IVC) (A-D) without ectopic calcification. Representative photomicrographs for picrosirius red (A and E), Masson's trichrome (B and F), Hart's (C, G), and von Kossa (D and H) stainings. Scale bars: $100 \mu \mathrm{m}$. I, Collagen content was similar in both the TEVG and native IVC. 

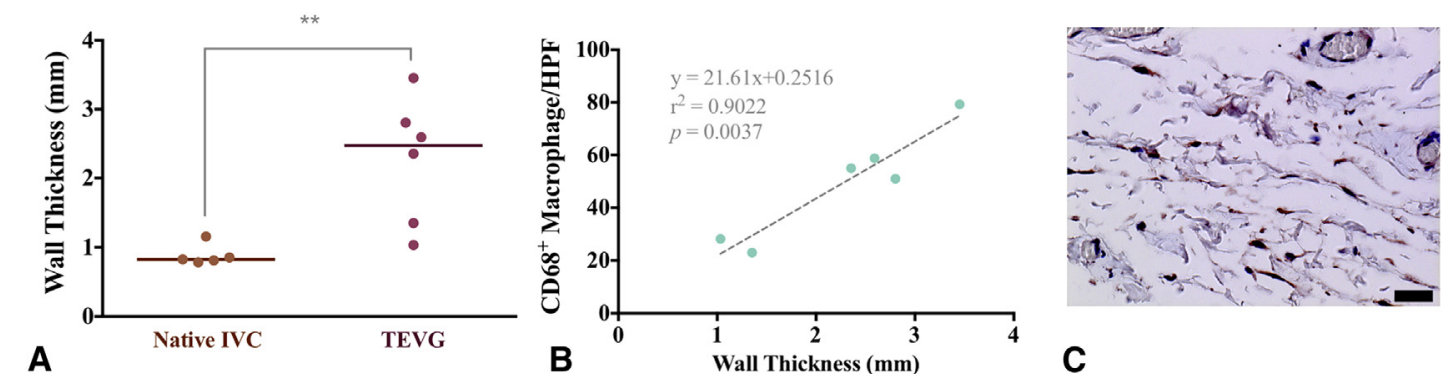

FIGURE 7. Macrophage analysis and histomorphometry. A, Histomorphometric comparison of the wall thickness between native inferior vena cava (IVC) and patient-specific nanofiber tissue-engineered vascular graft $(T E V G)$ revealed a statistically significant difference. $* * P=.0091$. B, In addition, there was a significant positive correlation between the wall thickness of patient-specific nanofiber TEVGs and CD68 ${ }^{+}$macrophages/high-power field $(H P F)$ in the patient-specific nanofiber TEVGs. $P=.0037$. C, Macrophage infiltration into the TEVGs was assessed by manual quantification of CD68 ${ }^{+}$cells. $S c a l e$ bar: $20 \mu \mathrm{m}$.

resulted in increased wall thickness of the TEVG (Figure 7, A).

\section{DISCUSSION}

The results of this study validate the efficacy of patient-specific, cell-free nanofiber TEVGs created by computer-aided design modeling, 3D printing, and electrospinning. The patient-specific TEVG was comparable to native IVC in terms of mechanical properties, angiography, histology, and immunohistochemistry. Serial angiography revealed that the initial pressure gradients between the cell-free, patient-specific TEVG and native IVC gradually resolved during the study's 6-month time course as the polymer scaffold was resorbed. In addition, the mechanical profile of the TEVG resembled that of the native IVC by the study endpoint.

Advances in 3D printing technologies are being increasingly integrated with the fields of regenerative and translational medicine to create novel solutions to challenges in tissue engineering. One example of this is the clinical use and development of a 3D-printed biodegradable tracheal splint. ${ }^{12}$ The relevance of 3D-printing technologies in clinical applications of TEVGs has been limited, however, owing to a lack of suitable materials that can be 3D-printed. Recent studies have focused on fully synthetic biodegradable polymers or synthetic polymers blended with natural proteins, such as collagen, elastin, gelatin, and chitosan. ${ }^{13-16}$ Although more current bioprinting efforts have produced biological blood vessels, ${ }^{17-19}$ the construct's mechanical properties are insufficient unless the tissue is further cultured for maturation. Previous studies also have examined TEVG fabrication using solvent-cast molding processes with synthetic biodegradable polymers, but 3D-printing TEVG scaffolds would best streamline the process of creating a patient-specific conduit. ${ }^{20,21}$

We recently demonstrated the functionality of a 3D-printed TEVG scaffold in a mouse model. The TEVG was an unseeded, cell-free construct and had adequate mechanical properties capable of supporting vascular tissue growth both in vitro and in vivo. ${ }^{22}$ The 3D-printed TEVGs were implanted as IVC conduits in immunocompromised mice and demonstrated biocompatibility and long-term efficacy at 1 year after the operation; however, the degradation rate of this scaffold [poly(propylene fumarate)] was suboptimal. In this work, we used Food and Drug Administration-approved materials with a known degradation profile, a 3D-printed mandrel, and electrospinning to produce custom TEVGs that could be easily translated to clinical application. Electrospinning is advantageous in that it is a highly tunable process by which a wide variety of polymer types and fiber sizes can be spun into various shapes of mandrels, thereby allowing for the rational design of custom-made scaffolds for tissue engineering. ${ }^{23}$

The current "one-size-fits-all" paradigm inadequately addresses the variable and complex anatomies present in the congenital heart disease population. Specifically, patient-specific TEVGs are useful for patients with single-ventricle anomalies who undergo extracardiac TCPC. Clinical studies have demonstrated that the geometry of the TCPC is a key factor in energy losses from suboptimal hepatic flow distribution. In addition, advances in computational models have led to the development of more physiologically realistic patientspecific simulations. ${ }^{24}$ Computational modeling has indicated that TCPC energy loss inversely correlates with decreased diameter of the TCPC conduit and pulmonary artery. These simulations also found correlations between hepatic flow distribution and caval offset, pulmonary flow distribution, and the connection angle between the TCPC and SVC. ${ }^{25}$ Further studies have examined Y-Fontan grafts to avoid flow collision from the SVC and IVC and promote equally distributed hepatic flow to the lungs. ${ }^{26}$ Although these studies may be limited by their retrospective nature, the number of institutions involved, and hypothetical computational simulations of rigid vessel walls, our 
patient-specific concept is able to address or altogether eliminate several of the concerns raised regarding traditional approaches to the Fontan circulation. The nanofiber construct evaluated in this report promotes autologous vessel growth with the potential of overcoming concerns associated with more rigid materials, such as flow and diameter mismatch. Patient-specific grafts can also avoid the need to offset SVC positioning in patients with limited implantation space owing to anatomic restrictions. Therefore, the patient-specific TEVG, by taking advantage of recent advances in imaging and computational modeling, provides immense potential to provide improved clinical and surgical outcomes. We suggest that our data support the continued pursuit of creating a complex, bifurcated, personalized TEVG for use as an optimized TCPC connection.

A patient-specific nanofiber scaffold is perhaps the next natural evolution of the TEVG. TEVGs in the form of biodegradable scaffold (woven PGA reinforced with PLCL) have been safely applied in low-pressure environments for patients with congenital heart disease undergoing extracardiac TCPC. ${ }^{4-6}$ Using routine radiographic modalities for TEVG observation, long-term follow-up demonstrated no instances of graft-related mortality and identified no cases of aneurysm formation, graft rupture, or ectopic calcification. ${ }^{27}$ Although the choice of PLCL and PGA as synthetic biodegradable materials has led to effective graft mechanical properties and remodeling in the previous human trial, we are currently evaluating second-generation electrospun PGA/PLCL and $\mathrm{PCL} /$ chitosan $^{28}$ scaffold designs with optimized nanofiber parameters that do not require cell seeding.

The patient-specific nanofiber TEVG displayed satisfactory vascular remodeling at 6 months postoperation. To the best of our knowledge, we are the first to demonstrate vascular SMC maturation in a biodegradable electrospun nanofiber scaffold in a sheep model. Compared with native IVC, the TEVG at 6 months had a significantly thicker layer of SMCs. Angiography showed significantly lower pressure gradients of the TEVG at 6 months than at 3 months. In addition, there was no significant difference at 6 months between the TEVG and native IVC in terms of burst pressure and compliance. A well-organized vascular SMC layer is required to prevent vascular calcification, but excessive SMC proliferation may eventually lead to stenosis or complete occlusion owing to hyperplasia. The 6-month time course of this study makes it difficult to ascertain whether the vascular SMC layer that developed in the TEVG would eventually resemble native IVC or progress to become occlusive stenosis at some later time point.

With regard to an adverse immune response at 6 months, we found a significant positive correlation between TEVG wall thickness and $\mathrm{CD}^{+} 8^{+}$macrophage infiltration into the scaffold. We previously reported that a scaffold seeded with bone marrow mononuclear cells transforms into a living vascular conduit with the ability to grow, repair, and remodel via an inflammation-mediated process. ${ }^{29}$ Additionally, our previous studies suggested that excessive macrophage infiltration contributes to occlusive vascular neotissue hyperplasia. ${ }^{30,31}$ Compared with native IVC, TEVG wall thickness was thicker, which may indicate the progression of chronic intimal hyperplasia.

Although the results of this study are promising and clearly encourage further investigation, this study was limited by its short in vivo time course and small sample size. Although creating a single ventricular model is more challenging in vivo, as is done clinically with the extracardiac TCPC procedure, future studies should be performed with complex anatomies. In addition, even though our scaffold demonstrated almost complete degradation in our previous in vivo studies, the degradation profile of the nanofiber TEVG should be further evaluated

\section{Background}

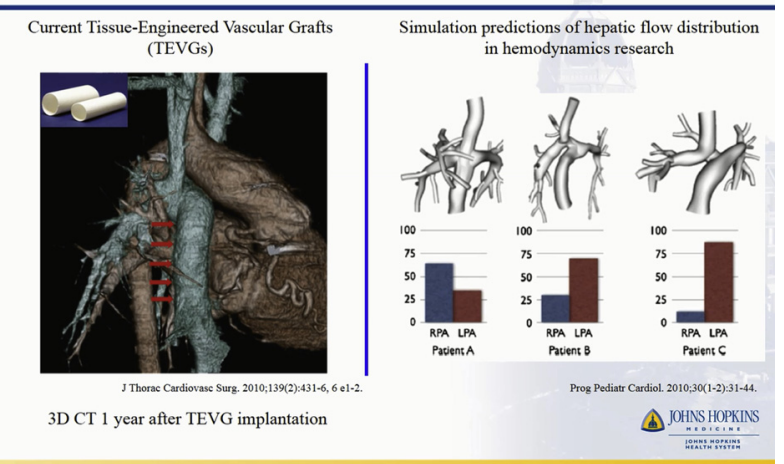

VIDEO 1. Preclinical study of patient-specific cell-free nanofiber tissueengineered vascular grafts (TEVGs) using 3D printing in a sheep model. Despite advances in surgical management of congenital heart disease, significant morbidity and mortality arise due to surgical complexity. Patientspecific graft design before surgery has the potential to overcome these challenges and improve patients' quality of life. This study demonstrates our integrated approach of 3D imaging, 3D printing, and tissue engineering technology to design and create patient-specific vascular grafts before surgery in a sheep model. There were no significant differences in burst pressure and compliance between grafts and native inferior vena cava (IVC). The grafts showed native-like neotissue formation at 6 months after surgery with their scaffolds almost fully degraded, with only $2 \%$ of the initial scaffolds remaining on average. Extracellular matrix, elastin, and collagen production and endothelialization were similar in grafts and native IVC, with no vascular calcification. The presence of multilayer smooth muscle cells and a decreasing pressure gradient at 6 months compared with 3 months suggested graft remodeling. There was a significant positive relationship between graft wall thickness and $\mathrm{CD}^{+} 8^{+}$macrophage infiltration into the scaffold. We have demonstrated the feasibility of creating patientspecific nanofiber TEVGs using combined technology of electrospinning and 3D printing. Video available at: http://www.jtcvsonline.org/article/ S0022-5223(16)31466-0/addons. 
during long-term follow up before being applied to human clinical trials. Despite our study limitations, patient-specific nanofiber TEVGs are an attractive alternative to prosthetic vascular grafts, such as Gore-Tex and Dacron, and our patient-specific nanofiber TEVG warrants further investigation owing to its immense clinical potential (Video 1).

\section{Conflict of Interest Statement}

Authors have nothing to disclose with regard to commercial support.

\section{References}

1. Dearani JA, Danielson GK, Puga FJ, Schaff HV, Warnes CW, Driscoll DJ, et al. Late follow-up of 1095 patients undergoing operation for complex congenital heart disease utilizing pulmonary ventricle to pulmonary artery conduits. Ann Thorac Surg. 2003;75:399-410; discussion 410-1.

2. Petrossian E, Reddy VM, McElhinney DB, Akkersdijk GP, Moore P, Parry AJ, et al. Early results of the extracardiac conduit Fontan operation. J Thorac Cardiovasc Surg. 1999;117:688-96.

3. Itatani K, Miyaji K, Tomoyasu T, Nakahata Y, Ohara K, Takamoto S, et al. Optimal conduit size of the extracardiac Fontan operation based on energy loss and flow stagnation. Ann Thorac Surg. 2009;88:565-72; discussion 572-3.

4. Naito Y, Imai Y, Shin'oka T, Kashiwagi J, Aoki M, Watanabe M, et al. Successful clinical application of tissue-engineered graft for extracardiac Fontan operation. J Thorac Cardiovasc Surg. 2003;125:419-20.

5. Shin'oka T, Matsumura G, Hibino N, Naito Y, Watanabe M, Konuma T, et al. Midterm clinical result of tissue-engineered vascular autografts seeded with autologous bone marrow cells. J Thorac Cardiovasc Surg. 2005;129:1330-8.

6. Hibino N, McGillicuddy E, Matsumura G, Ichihara Y, Naito Y, Breuer C, et al. Late-term results of tissue-engineered vascular grafts in humans. J Thorac Cardiovasc Surg. 2010;139:431-6. e1-2.

7. Johnson J, Ohst D, Groehl T, Hetterscheidt S, Jones M. Development of novel, bioresorbable, small-diameter electrospun vascular grafts. J Tissue Sci Eng. $2015 ; 6: 151$.

8. Raghavan ML, Vorp DA. Toward a biomechanical tool to evaluate rupture potential of abdominal aortic aneurysm: identification of a finite strain constitutive model and evaluation of its applicability. J Biomech. 2000;33: 475-82.

9. Bergmeister H, Schreiber C, Grasl C, Walter I, Plasenzotti R, Stoiber M, et al. Healing characteristics of electrospun polyurethane grafts with various porosities. Acta Biomater. 2013;9:6032-40.

10. Vorp DA, Schiro BJ, Ehrlich MP, Juvonen TS, Ergin MA, Griffith BP. Effect of aneurysm on the tensile strength and biomechanical behavior of the ascending thoracic aorta. Ann Thorac Surg. 2003;75:1210-4.

11. Lattouf R, Younes R, Lutomski D, Naaman N, Godeau G, Senni K, et al. Picrosirius red staining: a useful tool to appraise collagen networks in normal and pathological tissues. J Histochem Cytochem. 2014;62:751-8.

12. Zopf DA, Hollister SJ, Nelson ME, Ohye RG, Green GE. Bioresorbable airway splint created with a three-dimensional printer. N Engl J Med. 2013; 368:2043-5.

13. Brennan MP, Dardik A, Hibino N, Roh JD, Nelson GN, Papademitris X, et al. Tissue engineered vascular grafts demonstrate evidence of growth and development when implanted in a juvenile animal model. Ann Surg. 2008;248: 370-7.

14. Wu W, Allen RA, Wang Y. Fast-degrading elastomer enables rapid remodeling of a cell-free synthetic graft into a neoartery. Nat Med. 2012;18:1148-53.
15. Zhou M, Qiao W, Liu Z, Shang T, Qiao T, Mao C, et al. Development and in vivo evaluation of small-diameter vascular grafts engineered by outgrowth endothelial cells and electrospun chitosan/poly( $\varepsilon$-caprolactone) nanofibrous scaffolds. Tissue Eng Part A. 2014;20:79-91.

16. Mrówczynski W, Mugnai D, de Valence S, Tille JC, Khabiri E, Cikirikcioglu M, et al. Porcine carotid artery replacement with biodegradable electrospun poly-e-caprolactone vascular prosthesis. J Vasc Surg. 2014;59:210-9.

17. Skardal A, Zhang J, Prestwich GD. Bioprinting vessel-like constructs using hyaluronan hydrogels crosslinked with tetrahedral polyethylene glycol tetracrylates. Biomaterials. 2010;31:6173-81.

18. Yu Y, Zhang Y, Martin JA, Ozbolat IT. Evaluation of cell viability and functionality in vessel-like bioprintable cell-laden tubular channels. J Biomech Eng. 2013;135:91011.

19. Itoh M, Nakayama K, Noguchi R, Kamohara K, Furukawa K, Uchihashi K, et al. Scaffold-free tubular tissues created by a bio-3D printer undergo remodeling and endothelialization when implanted in rat aortae. PLoS One. 2015;10: e0136681.

20. Nelson GN, Mirensky T, Brennan MP, Roh JD, Yi T, Wang Y, et al. Functional small-diameter human tissue-engineered arterial grafts in an immunodeficient mouse model: preliminary findings. Arch Surg. 2008;143:488-94.

21. Melchiorri AJ, Hibino N, Brandes ZR, Jonas RA, Fisher JP. Development and assessment of a biodegradable solvent cast polyester fabric small-diameter vascular graft. J Biomed Mater Res A. 2014;102:1972-81.

22. Melchiorri AJ, Hibino N, Best CA, Yi T, Lee YU, Kraynak CA, et al. 3D-printed biodegradable polymeric vascular grafts. Adv Healthc Mater. 2016;5:319-25.

23. Rocco KA, Maxfield MW, Best CA, Dean EW, Breuer CK. In vivo applications of electrospun tissue-engineered vascular grafts: a review. Tissue Eng Part B Rev. 2014;20:628-40.

24. de Zélicourt DA, Marsden A, Fogel MA, Yoganathan AP. Imaging and patient-specific simulations for the Fontan surgery: current methodologies and clinical applications. Prog Pediatr Cardiol. 2010;30:31-44.

25. Tang E, Restrepo M, Haggerty CM, Mirabella L, Bethel J, Whitehead KK, et al. Geometric characterization of patient-specific total cavopulmonary connections and its relationship to hemodynamics. JACC Cardiovasc Imaging. 2014;7: 215-24.

26. Restrepo M, Crouch AC, Haggerty CM, Rossignac J, Slesnick TC, Kanter KR, et al. Hemodynamic impact of superior vena cava placement in the Y-graft Fontan connection. Ann Thorac Surg. 2016;101:183-9.

27. Kurobe H, Maxfield MW, Breuer CK, Shinoka T. Concise review: tissue-engineered vascular grafts for cardiac surgery: past, present, and future. Stem Cells Transl Med. 2012;1:566-71.

28. Fukunishi T, Best CA, Sugiura T, Shoji T, Yi T, Udelsman B, et al. Tissueengineered small diameter arterial vascular grafts from cell-free nanofiber PCL/chitosan scaffolds in a sheep model. PLoS One. 2016;11:e0158555.

29. Roh JD, Sawh-Martinez R, Brennan MP, Jay SM, Devine L, Rao DA, et al. Tissue-engineered vascular grafts transform into mature blood vessels via an inflammation-mediated process of vascular remodeling. Proc Natl Acad Sci U S A. 2010;107:4669-74.

30. Hibino N, Villalona G, Pietris N, Duncan DR, Schoffner A, Roh JD, et al. Tissue-engineered vascular grafts form neovessels that arise from regeneration of the adjacent blood vessel. FASEB J. 2011;25:2731-9.

31. Hibino N, Mejias D, Pietris N, Dean E, Yi T, Best C, et al. The innate immune system contributes to tissue-engineered vascular graft performance. FASEB J. 2015;29:2431-8.

Key Words: 3D printing, cell-free tissue engineering, congenital heart disease, electrospun nanofibers, Fontan circulation, patient-specific, preclinical study, sheep model, tissue-engineered vascular graft 